\title{
Theory of rare gas alloys: heat capacity
}

\author{
M.I. Bagatskii, S.B. Feodosyev, I.A. Gospodarev, O.V. Kotlyar, E.V. Manzhelii, \\ A.V. Nedzvetskiy, and E.S. Syrkin \\ B. Verkin Institute for Low Temperature Physics and Engineering of the National Academy of Sciences of Ukraine \\ 47 Lenin Ave., Kharkov 61103, Ukraine \\ E-mail: feodosiev@ilt.kharkov.ua
}

Received October 20, 2006

\begin{abstract}
Low-temperature heat capacity of cryocrystals, which contain impurity clusters has been investigated theoretically and experimentally. Such defects might essentially enrich low-frequency part of the phonon spectrum by introducing both localized and delocalized vibrations. The effect of both types of the vibrations on the temperature dependence of the heat capacity is analyzed. Heat capacity of the disordered solid solution $\mathrm{Kr}-\mathrm{Ar}$ (Ar concentration is $\sim 25 \%$ ) is studied as an example of the effect of the light weakly connected impurities on the low-temperature thermodynamic characteristics of the system. The mass defect of such an impurity induces «phonon pumping» from the low-frequency part of the spectrum into the high-frequency part and decreasing the low-temperature heat capacity, while the weakened interaction between the impurity and the host atoms combined with even weaker interaction between the impurities leads to the formation of the low-temperature maximum on the heat capacity temperature dependence. The analysis performed shows that at rather high Ar concentrations, the nonmonotonous temperature dependence of the relative change in the heat capacity of solid $\mathrm{Kr}_{1-p} \mathrm{Ar}_{p}$ solutions is determined by excitation of delocalized high-dispersion low-frequency phonons.
\end{abstract}

PACS: 63.20.-e Phonons in crystal lattice;

63.20.Mt Phonon-defect interaction;

63.50.+x Vibrational states of disordered systems;

63.70.+h Statistical mechanics of lattice vibrations and displacive phase transitions.

Keywords: disordered solid solution, impurity cluster, boson peak, Jacoby matrix, Green function.

\section{Introduction}

For many years the influence of defects upon the physical properties of cryocrystals has been one of the most important problems of low temperature physics of solids that stimulated intense theoretical and experimental research (e.g., see monograph [1] and references therein). The results obtained on such objects are explainable with high accuracy within quite simple crystal lattice models and can be generalized for a very wide class of crystalline structures.

At present there is a practically completed theory interpreting the variations of the crystal properties caused by the so-called isolated defects, whose influence upon one another is negligible. It is certainly interesting to investigate crystals with defects that can be defined as complex ones, for example, impurities located near a sample boundary or a vacancy. When the concentration of impurity atoms in the lattice grows, complex defects can ap- pear in addition to the isolated ones. Complex defects are formed by closely-spaced impurities. In some cases they may be considered as isolated defects [2-5] and can be described in terms of the regular perturbation theory.

Special interest is focused on systems consisting of a cryocrystalline matrix containing randomly-dissolved impurity atoms. At a growing concentration the spectral characteristics of such solutions exhibit properties that are typical of amorphous compounds, glasses, biopolymers and so on. One of the features characterizing such systems is the anomalous frequency distribution of phonons in the long-wavelength (low-frequency) region. This is evident in Raman and neutron scattering experiments [6-8] and in the behavior of the low-temperature heat capacity and thermal conductivity [9-11].

This anomaly of the frequency distribution of phonons can be described as a maximum of the magnitude $\mathrm{I} \equiv g(\omega) / \omega^{2}$, where $g(\omega)$ is the phonon density of states and $\omega$ is the frequency. The maximum was called a «boson 
peak». It is typical for glasses, amorphous media, and other disordered systems in which the influence of defects goes beyond local disturbances. The change in the vibrational and other physical properties of such systems cannot be interpreted as regular degenerate perturbation.

In this study we investigate theoretically how the lowtemperature heat capacity of solid $\mathrm{Kr}_{1-p} \mathrm{Ar}_{p}$ solutions changes at growing concentration $p$ of Ar atoms. Argon and krypton are highly soluble in each other and the parameter $p$ can take any value varying from zero to unity [12]. The calculated results are compared with the currently available experimental data. It is shown that the change in the low-temperature heat capacity at $p \gtrsim 20 \%$ and lower $(\sim 5-10 \%)$ concentrations of argon has qualitative features induced by delocalized excitations analogous with boson peaks that appear in addition to the local (including complex) defects.

\section{Changes in heat capacity of krypton caused by argon impurity}

We develop the model crystal lattice of the $\mathrm{Kr}_{1-p} \operatorname{Ar}_{p}$ system on the basis of the fcc structure with the lattice parameter of pure krypton ( $a \approx 5.59 \AA$ [1]). In our description the interatomic interaction in solidified inert gases is reduced to the interaction between the nearest neighbors. In the general case this interaction is characterized by three force constants $\alpha, \beta$, and $\gamma$ :

$$
\Phi_{i k}\left(\frac{a}{2} ; \frac{a}{2} ; 0\right)=\left(\begin{array}{ccc}
\alpha & \gamma & 0 \\
\gamma & \alpha & 0 \\
0 & 0 & \beta
\end{array}\right) .
$$

The other force constant matrices can be found from Eq. (1) by $O_{h}-$ group symmetry operations, and the matrix of self-action is

$$
\Phi_{i k}(0 ; 0 ; 0)=(8 \alpha+4 \beta) \delta_{i k} .
$$

Since a cubic-symmetry crystal has three independent elastic moduli $C_{11}, C_{12}$, and $C_{66}$, we can use their values and estimate unambiguously the force constants $\alpha, \beta$, and $\gamma$. For krypton [13] we obtain $\alpha_{\mathrm{Kr}-\mathrm{Kr}}=7260.3 \mathrm{dyn} / \mathrm{cm}$, $\beta_{\mathrm{Kr}-\mathrm{Kr}}=310.75 \mathrm{dyn} / \mathrm{cm}, \gamma_{\mathrm{Kr}-\mathrm{Kr}}=7797.0 \mathrm{dyn} / \mathrm{cm}$. The force constants characterizing the $\mathrm{Kr}-\mathrm{Ar}$ and $\mathrm{Ar}-\mathrm{Ar}$ interactions can be calculated with help of the LennardJones potentials (parameters $\varepsilon$ and $\sigma$ for $\mathrm{Kr}$ and $\mathrm{Ar}$ see e.g. [1]):

$$
\begin{gathered}
\alpha_{\mathrm{Kr}-\mathrm{Ar}}=7804.0, \beta_{\mathrm{Kr}-\mathrm{Ar}}=127.00, \gamma_{\mathrm{Kr}-\mathrm{Ar}}=7310.0 ; \\
\alpha_{\mathrm{Ar}-\mathrm{Ar}}=1551.83, \beta_{\mathrm{Ar}-\mathrm{Ar}}=220.677, \gamma_{\mathrm{Ar}-\mathrm{Ar}}=1331.15 .
\end{gathered}
$$

We assume that the Ar-Ar interaction is the same for the nearest Ar pairs and the larger clusters of defects (triangles, tetrahedrons, etc.) that can form as the Ar concentration increases.
The calculations performed in this study are based on the $\mathcal{J}$-matrix technique (e.g., see [14-16]). The method does not use explicitly the translational symmetry of the crystal lattice and permits a straightforward calculation of the spectral densities corresponding to the displacements of different atoms of the system along displacements of different crystallographic directions $i$ :

$$
\rho_{i}(\omega, \mathbf{r})=\frac{1}{\pi} \operatorname{Im} \mathcal{G}_{i}(\omega, \mathbf{r}) .
$$

Here $\mathbf{r}$ is the radius-vector characterizing the position of the particular atom, $\omega$ is the frequency; the Green function $\mathcal{G}_{i}(\omega, \mathbf{r})$ is expressed in terms of the matrix element of operator-resolvent as follows:

$$
\mathcal{G}_{i}(\omega, \mathbf{r})=2 \omega \cdot\left\langle\begin{array}{c}
\mathbf{r} \\
u_{i}
\end{array}\left|\left(\omega^{2} \hat{\mathcal{I}}-\hat{\mathcal{L}}\right)^{-1}\right| \begin{array}{c}
\mathbf{r} \\
u_{i}
\end{array}\right\rangle
$$

where the symbol $\left\langle\mathbf{u}_{i}\right|$ has the meaning of the displacement of the atom with the radius-vector $\mathbf{r}$ along the crystallographic direction $i$ (a certain vector in the space of atom displacements in the system $H$ ); $\hat{\mathcal{I}}$ is the unit operator, $\hat{\mathcal{L}}$ is the operator describing the atomic oscillations in the system, its eigenvalues are squared eigenfrequencies. The matrix of this operator can be expressed in terms of the force constant matrix and the masses of the interacting atoms $m(\mathbf{r})$ and $m\left(\mathbf{r}^{\prime}\right)$.

The spectral density of the system is

$$
\langle\rho(\omega, p)\rangle=\lim _{N \rightarrow \infty} \frac{2 \omega}{\pi N} \operatorname{Sp} \operatorname{Im}\left(\omega^{2} \hat{\mathcal{I}}-\hat{\mathcal{L}}\right)^{-1},
$$

According to [17-20], it is a self-averaging quantity and can be estimated through averaging the functions over all r-positions and displacement directions $i$.

The random distribution of the Ar impurity atoms was performed using a random-number generator of pseudorandom numbers distributed uniformly in the interval $(0,1)$. The random-number generator operates on the multiplying congruent principle [21]. We calculated the spectral densities $\langle\rho(\omega, p)\rangle$ for different concentration of impurity atoms. At each concentration, the averaging was made over several thousands of random configurations of the impurity distribution. For each configuration the density of states was determined through averaging over several tens of spectral densities corresponding to the displacements along different crystallographic directions of several tens of sequentially arranged atoms.

The vibrational heat capacity $C_{v}$ (heat capacity at constant volume) is expressed in terms of the phonon density as (e.g., see [22]) of solid solutions is expressed in terms of $v(\omega)$ as

$$
C_{v}(T, p)=3 R \int_{\mathcal{D}}\left(\frac{\hbar \omega}{k T}\right)^{2} \sinh ^{-2}\left(\frac{\hbar \omega}{k T}\right) v(\omega) d \omega,
$$


where the integral is calculated in the whole region of the atomic oscillation frequencies $\mathcal{D}$ both over the quasi-continuous spectrum band and over the discrete levels (if there are).

We obtain $v(\omega)=\left\langle\rho_{i}(\omega, p)\right\rangle$ for solid solutions and $v(\omega)=\rho_{i}(\omega, \forall \mathbf{r})$ for a perfect single-atom crystalline lattice of cubic symmetry. The function $\rho_{\forall i}(\omega, \forall \mathbf{r})$ is the spectral density caused by the displacement of an arbitrary atom in any of the crystallographic directions.

It is known that at low concentrations the interaction between the impurity atoms is negligible. It is also assumed that the impurity-induced change in the additive thermodynamic characteristics is linear in impurity concentration $p$. Thus, at $p<<1$

$$
\frac{\Delta C_{v}(T, p)}{C_{v}^{(\mathrm{Kr})}(T)} \approx p \frac{\Delta C_{v}^{l}(T)}{C_{v}^{(\mathrm{Kr})}(T)},
$$

where $\Delta C_{v}^{l}(T)$ is the change in the heat capacity per local defect. The disturbance of the lattice vibrations generated by a local defect is as a rule, localized around this defect.

The $\Delta C_{v}^{l}(T)$-value can be calculated using, for example, a shift function if the disturbance is regular and degenerate $[23,24]$. According to the traditional interpretation of crystal lattice vibrations as a superposition of plane waves, only the disturbance generated by an isotopic impurity can be considered as degenerate (in three-dimensional vector models). The $\mathcal{J}$-matrix method treats the disturbance as degenerate if it is generated by an impurity with changed force constants in the case of the noncentral interaction between the atoms [25]. The presence of the noncentral forces allows the disturbance to be degenerated only in the subspace that can be transformed in one-dimensional representations of the symmetry group of the particular lattice.

At the same time, local disturbances do not affect the band width of the quasi-continuous spectrum of the crystal. They can only form discrete local levels beyond the band. Therefore, the asymptotic behavior of the $\mathcal{J}$-matrix elements representing the operators $\hat{\mathcal{L}}$ that describe the lattice vibrations do not change under the influence of a local defect. It is natural to call such disturbances asymptotically degenerate.

Since the arbitrary matrix element $\mathcal{G}_{m n}\left(\omega^{2}\right)$ of the resolvent operator $\hat{\mathcal{G}}\left(\omega^{2}\right) \equiv\left(\omega^{2} \hat{\mathcal{I}}-\hat{\mathcal{L}}\right)^{-1}$ can be expressed strait forwardly through the element $\mathcal{G}_{00}\left(\omega^{2}\right) \equiv \mathcal{G}\left(\omega^{2}\right)$ (Green function), for $m<n$ we obtain [5]:

$$
\begin{aligned}
& \mathcal{G}_{m n}(\lambda) \equiv\left(\mathbf{h}_{m}, \hat{\mathcal{G}}(\lambda) \mathbf{h}_{n}\right)= \\
& =-\mathcal{P}_{m}(\lambda) \mathcal{Q}_{n}(\lambda)+\mathcal{P}_{m}(\lambda) \mathcal{P}_{n}(\lambda) \mathcal{G}_{00}(\lambda),
\end{aligned}
$$

then the change in the heat capacity for such asymptotically degenerated local disturbance can be written in the form of the integral of Eq. (6) with the function $v(\omega)$ substituted by

$$
\begin{aligned}
\Delta v(\omega)= & 2 \omega \sum_{n=0}^{r \rightarrow \infty}\left[\widetilde{\mathcal{P}}_{n}^{2}\left(\omega^{2}\right) \tilde{\rho}\left(\omega^{2}\right)-\mathcal{P}_{n}^{2}\left(\omega^{2}\right) \rho\left(\omega^{2}\right)\right]+ \\
& +\sum_{l}^{n} \delta\left(\omega-\omega_{l}\right) .
\end{aligned}
$$

The second sum in Eq. (8) describes the contribution of local frequencies if they appear in the disturbed system; $\mathcal{P}_{n}\left(\omega^{2}\right)$ are the Jacobian matrix-generated polynomials (e.g., see [14-16]); the tilded variables refer to a disturbed system and can be calculated using the corresponding Jacobian matrix; the nontilded variables describe an ideal perfect lattice. The accuracy of computation by Eqs. (6), (8) using the finite rank $\mathcal{J}$-matrix corresponds to the accuracy of calculation of the perfect-lattice heat capacity with the aid of the matrix of the same rank.

\section{Results and discussion}

By the methods above mentioned we received the following results. Figure 1 illustrates the temperature dependencies of the relative change

$$
\frac{\Delta C_{v}(T)}{C_{v}^{(\mathrm{Kr})}(T)} \equiv \frac{\left\langle C_{v}(T, p)\right\rangle-C_{v}^{(\mathrm{Kr})}(T)}{C_{v}^{(\mathrm{Kr})}(T)}
$$

in the heat capacity of solid $\mathrm{Kr}_{1-p} \operatorname{Ar}_{p}$ solutions with various $\mathrm{Ar}$ concentrations $p$ in reference to the heat capacity of pure Kr.

The dependence is smooth at moderate $p$-values ( 5 and $10 \%$; curves 1 and 2 , respectively). At $p=15 \%$ (curve 3 ) the dependence exhibits some «flattening» in the interval $3.5 \mathrm{~K} \leq T \leq 10 \mathrm{~K}$. On a further increase in the Ar concentration: $p=24.4 \%$ (curve 4 ) and $50 \%$ (curve 5), two extrema appear in $\Delta C_{v}(T, p) / C_{v}^{(\mathrm{Kr})}(T)$ - a maximum at $T \approx 3.5 \mathrm{~K}$ and a minimum at $T \approx 9 \mathrm{~K}(24.4 \%)$ and $T \approx 12.5 \mathrm{~K}(50 \%)$.

The measured temperature dependence of the relative change in the heat capacity at $p=24.4 \%$ [26] (O) is

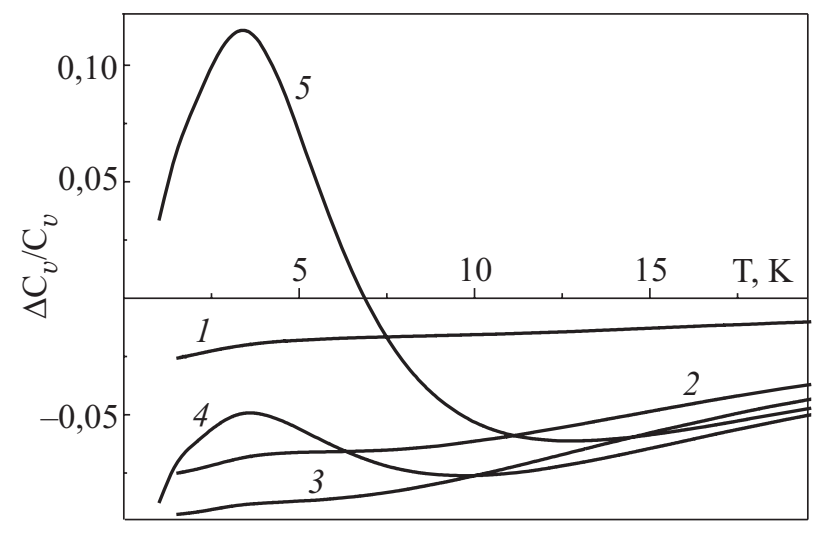

Fig. 1. Temperature dependences of the relative change in the heat capacity at a growing concentration of Ar impurity $p, \%$ : 5 (1), 10 (2), 15 (3), 24.4 (4), 50 (5). 


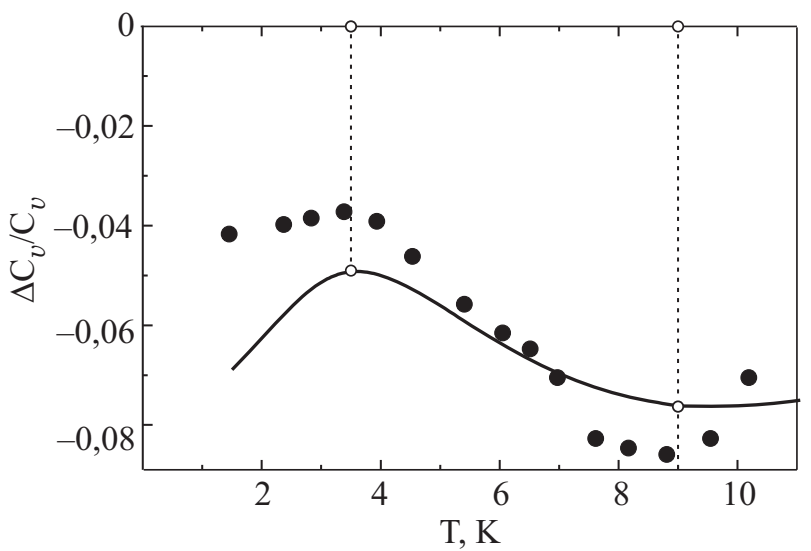

Fig. 2. Temperature dependences of the relative change in the heat capacity of solid $\mathrm{Kr}_{0.756} \mathrm{Ar}_{0.244}$ solution: theoretical calculation (-), experiment $(\bigcirc)$.

shown in Fig. 2. There are two extrema in this case too: a maximum at $T \approx 3.5 \mathrm{~K}$ and a minimum at $T \approx 9 \mathrm{~K}$. The solid line is a portion of the curve of Fig. 1, which illustrates a theoretical calculation of the same magnitude at the same concentration. It is seen that the experimental and theoretical results are in good agreement - the extrema temperatures coincide with a high degree of accuracy and the deviations of $\Delta C_{v}(T, p) / C_{v}^{(\mathrm{Kr})}(T)$ are actually within the accuracy of the experiment. True, the character of dilation at these $p$-values is more complicated than that allowed for in the model.

Let us analyze the possible reasons for the qualitative behavior of the relative change in the heat capacity at growing concentration.

Figure 3 illustrates the temperature dependencies of the $\Delta C_{v}^{1 \mathrm{Ar}}(T) / C_{v}^{(\mathrm{Kr})}(T)$ - parameter describing the change in the heat capacity of $\mathrm{Kr}$ caused by an isolated atom of the Ar impurity (curve 1) and the $\Delta C_{v}^{2 \mathrm{Ar}}(T) / C_{v}^{(\mathrm{Kr})}(T)-$ parameter describing the change in the heat capacity caused by an isolated pair of adjacent Ar atoms (curve 2). The smooth rise of curve 1 with temperature and the very weak temperature dependence of curve 2 permit us to explain the heat capacity variation at low (down to 10\%) concentrations of Ar impurity at the expense of a superposition of local disturbances localized near the defects* that generate them. This explanation is totally unsuitable for the double extrema behavior of the $\Delta C_{v}(T, p) / C_{v}^{(\mathrm{Kr})}(T)$ - parameter at higher concentrations.

Since in the $\mathrm{Kr}_{1-p} \operatorname{Ar}_{p}$ solutions the linkage between the Ar atoms is much weaker than the $\mathrm{Kr}-\mathrm{Kr}$ or $\mathrm{Kr}-\mathrm{Ar}$ bonds, at $p \gtrsim 20 \%$, when practically each impurity atom has identical impurity atoms among its nearest neighbors,

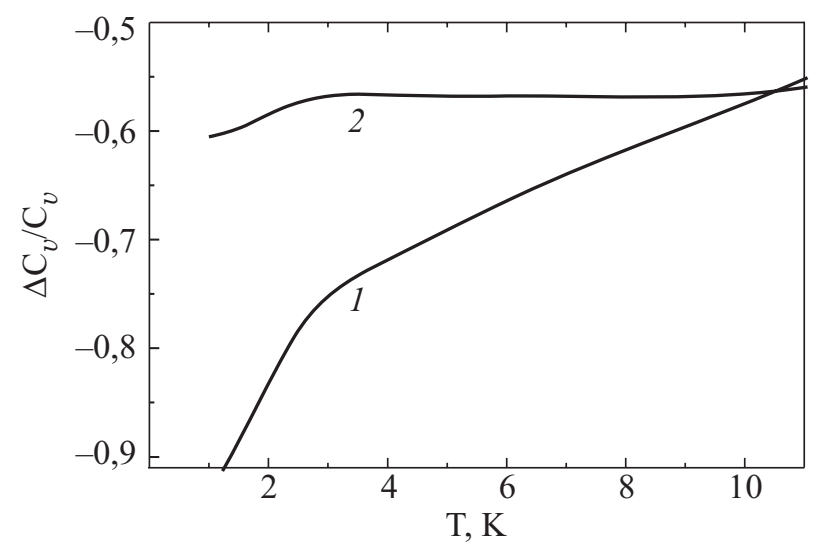

Fig. 3. Temperature dependences of the relative change in the heat capacity caused by substitution Ar impurity: curve 1 isolated $\mathrm{Ar}$ atom, curve $2-$ isolated pair of adjacent Ar atoms.

some quasi-continuous distribution of the weak bonds occurs in the lattice. In this case each region having the characteristic size $l \gtrsim \lambda$ (sound wavelength) can be characterized by its own set of elastic constants, its own longitudinal and transverse sound velocities which acquire a meaning of randomly distributed parameters.

No quasi-local low-frequency oscillations can occur in such systems. The low frequency regions of the density of states of the $\mathrm{Kr}_{1-p} \mathrm{Ar}_{p}$ systems are shown in Fig. 4, the Ar concentrations being as in Fig. 1 (the curves are numbered as in Fig. 1, the dashed line shows the density of states of ideal $\operatorname{Kr}[13])$. In the frequency interval $\omega \leq \omega *$ (the frequency of the first van Hove singularity) none of the curves has any sign of the characteristic quasi-local maximum. The curves have typical «quasi-Debye» shapes. In other words, the oscillations at these frequencies, which are responsible for the behavior of the heat capacity in the temperature interval $1-20 \mathrm{~K}$, are completely delocalized.

At the same time, such systems with randomly distributed force and elastic parameters are noted for more intensive sound wave dispersion. As a result, the curve $v(\omega)$ starts to deviate from the quadratic (Debye) behavior at lower temperatures. For this reason the ratio $v(\omega) / \omega^{2}$ reaches a maximum in glasses and some other disordered systems (e.g., the system in [27]). This is the so-called «boson maximum» (or «boson peak» $[6-11,27-32]$ ). Note that in these studies this peak was investigated mainly for the phonons with frequencies at which the sound velocity becomes dependent on the wave vector (from «propagons» to «diffusons», according to the terms of [33]). The absence of a distinct boundary between the propagons

* The contributions from impurity clusters such as equilateral triangles and tetrahedrons, are qualitatively similar to the contributions made by impurity pairs; besides, the concentration of such localized defects is extremely low. 


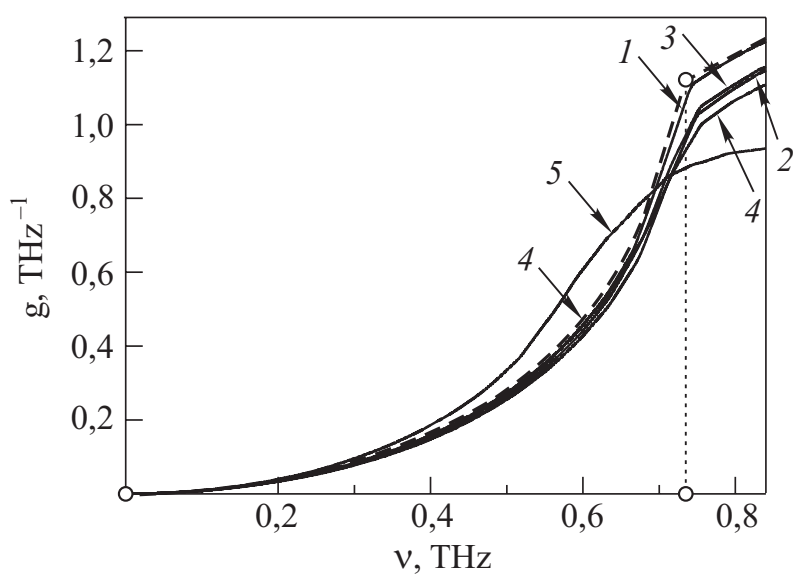

Fig. 4. Evolution of low frequency regions (until the first van Hove singularity appears - the thin dashed vertical line) of the phonon densities of the $\mathrm{Kr}_{1-p} \mathrm{Ar}_{p}$ solution at a growing Ar concentration $p$. Heavy dashed line - phonon density of pure Kr. The curves are numbered as in Fig. 1.

and diffusons is usually identified with the Ioffe-Regel effect (e.g., see [32,33]).

However, even higher-frequency acoustic phonons whose dispersion ceases being linear (the so-called «diffusons») can form a similar maximum if its frequency is not higher than $\omega *$.

Figure 5 illustrates the ratios $v(\omega) / \omega^{2}$ for the $\mathrm{Kr}_{1-p} \mathrm{Ar}_{p}$. There is a maximum only at $p=50 \%$. At other concentrations it has no time to form the maximum of the frequency of the first van Hove singularity -- opening of isofrequency surfaces of transverse acoustic oscillations. However, as soon as the propagation of the sound wave slowing down, the number of the low-frequency phonons (especially those with $k \rightarrow 0$ ) increases and so does the low temperature heat capacity. On the other hand, the number of the low frequency phonons decreases because of the small mass of the impurity atoms. It is natural that the two mechanisms - the increase and the decrease in the number of phonons-compete in $\mathrm{Ar}-\mathrm{Kr}$ solutions. Note that at $p=24.4 \%$ the maximum of the relative change in the heat capacity (Figs. 1,2) is negative and becomes positive only at higher Ar concentrations (e.g., see Fig. 1, curve 5).

Thus, the analysis performed shows that at rather high Ar concentrations, the nonmonotonous temperature dependence of the relative change in the heat capacity of solid $\mathrm{Kr}_{1-p} \operatorname{Ar}_{p}$ solutions is determined by excitation of delocalized high-dispersion low-frequency phonons. In turn, the high-dispersion low-frequency phonons are due to the random distribution of weak bonds in the lattice, which are typical for the Ar-Ar interaction in this solution. Note that the distributed weak bonds cause a severe local anisotropy of the oscillations both of Ar and $\mathrm{Kr}$ atoms. The local anisotropy leads to some pushing the

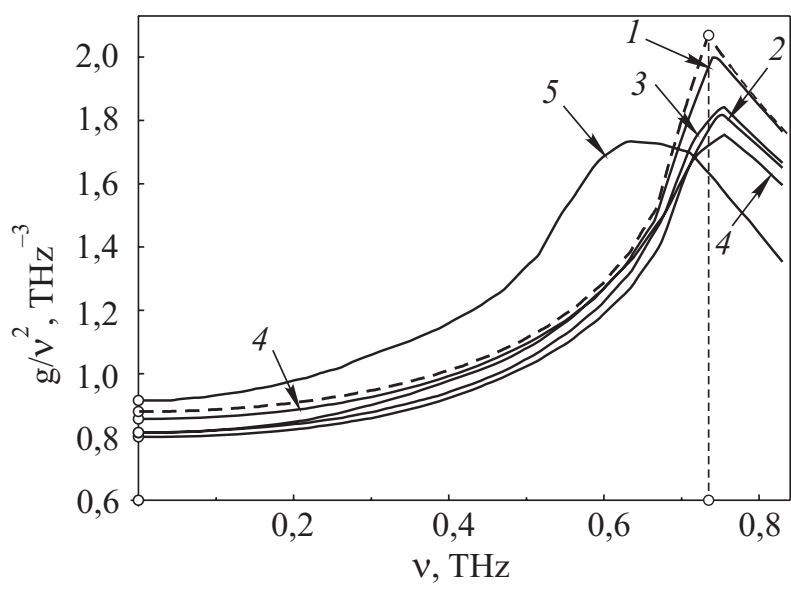

Fig. 5. Evolution of low-frequency $g(v) / v^{2}-$ regions at a growing $\mathrm{Ar}$ concentration $p$ in the solid $\mathrm{Kr}_{1-p} \mathrm{Ar}_{p}$ solution. (the notation is as in Fig. 4).

phonons towards the ends of the band of the quasi-continuous spectrum, which increases the number of low frequency phonons.

1. A.F. Prikhot'ko, V.G. Manzhelii, I.Ya. Fugol', Yu.B. Gaididei, I.N. Krupskii, V.M. Loktev, E.V. Savchenko, V.A. Slyusarev, M.A. Strzhemechny, Yu. Freiman, and I.I. Shansky, Cryocrystals, Naukova Dumka, Kiev (1983).

2. V.I. Peresada and E.S. Syrkin, Fiz. Tverd. Tela (Leningrad) 18, 336 (1976).

3. E.T. Bruk-Levinson, I.A. Gospodarev, A.I. Zakharov, and E.S. Syrkin, Izv. AN Bel. SSR 3, 78 (1988).

4. S.B. Feodosyev, I.A. Gospodarev, V.I. Grishaev, A.M. Kosevich, O.V. Kotlyar, and E.S. Syrkin, J. Low Temp. Phys. 139, 665 (2005).

5. O.V. Kotlyar and S.B. Feodosyev, Low Temp. Phys. 32, 256 (2006).

6. Amorphous Solids Low Temperature Properties, W.A. Phillips (ed.), Springer-Verlag, Berlin (1981).

7. A.P. Sokolov, A. Kisliuk, D. Quitmann, and E. Duval, Phys. Rev B48, 7692 (1998).

8. A.P. Sokolov, L. Rossler, A. Kisliuk, and D. Quitmann, Phys. Rev. Lett. 71, 2062 (1993).

9. V.K. Malinovsky, V.N. Novikov, P.P. Parshin, A.P. Sokolov, and M.G. Zemlyanov, Europhys. Lett. 11, 43 (1990).

10. C.C. Yu and J.J. Freeman, Phys. Rev B36, 7620 (1987).

11. W. Schrimacher, Europhys. Lett. 73, 892 (2006).

12. V.G. Manzhelii, A.I. Prokhvatilov, I.Ya. Minchina, and L.D. Yantsevich, Handbook of Binary Solutions of Cryocrystals, Begel house inc., New York, Wallingford (UK) (1996).

13. S.B. Feodosyev, I.A. Gospodarev, V.O. Kruglov, and E.V. Manzhelii, J. Low Temp. Phys. 139, 651 (2005).

14. V.I. Peresada, in: Condensed Matter Physics [in Russian], FTINT AN Ukr. SSR, Kharkov, 2, 172 (1968).

15. V.I. Peresada, V.N. Afanas'ev, and V.S. Borovikov, Low Temp.Phys. 1, 227 (1975). 
16. R. Haydock, in: Solid State Phys. 135, H. Ehrehreich et al. (eds.), Academic Press, New York (1980), p. 129.

17. I.M. Lifshitz, S.A. Gredeskul, and L.A. Pastur, Introduction to the Theory of Disordered Systems, Wiley-Interscience, New York (1988).

18. I.M. Lifshitz, JETP 44, 1723 (1963).

19. I.M. Lifshitz, Uspekhi Phis. Nauk 83, 617 (1964) [in Russian].

20. I.M. Lifshitz, S.A. Gredeskul, and L.A. Pastur, J. Stat. Phys. 38, 37 (1985).

21. P. L'ecuyer, Efficient and Portable Combined Random Number Generators, in: CACM 31, 6 (1988).

22. A. Maradudin, Solid State Phys. 18, 273 (1966); ibid. 19, 1 (1966).

23. I.M. Lifshitz, DAN SSSR 48, 83 (1945).

24. I.M. Lifshitz, JETP 17, 1076 (1948).

25. V.I. Peresada and V.P. Tolstoluzhskii, Sov. J. Low Temp. Phys. 3, 383 (1977).
26. M.I. Bagatskii, E.S. Syrkin, and S.B. Feodosyev, Sov. J. Low Temp. Phys. 18, 629 (1992).

27. V. Gurarrie and A. Atland, Phys. Rev. Lett. 94, 245502 (2005).

28. D.A. Parshin, Phys. Solid State 36, 991 (1994).

29. W. Schrimacher, G. Diezeman, and C. Ganter, Phys. Rev. Lett. 81, 136 (1998).

30. V.L. Gurevich, D.A. Parshin, and H.R. Schroder, Phys. Rev. B67, 094203 (2003).

31. A.I. Chumakov, I. Sergeev, U. van Burck, W. Schrimacher, T. Asthalter, R. Ruffer, O. Leupold, and W. Petry, Phys. Rev. Lett 92, 245508 (2004).

32. M.I. Klinger and A.M. Kosevich, Phys. Lett. A295, 31 (2002).

33. P.B. Allen, J.I. Feldman, J. Fabian, and F. Wooten, Philos. Mag. B72, 1715 (1999). 